\title{
Fractal dimensions of cave for exemplary gypsum cave-mazes of Western Ukraine
}

\author{
Viacheslav Andreychouk', Tomasz Błachowicz², Krzysztof Domino² \\ ${ }^{1}$ Faculty of Earth's Sciences, Silesian University, Sosnowiec, Poland, geo@wnoz.us.edu.pl \\ ${ }^{2}$ Institute of Physics - Center for Science and Education, Silesian University of Technology, Gliwice, Poland
}

\begin{abstract}
Gypsum labyrinthine caves are characterized by a complex spatial structure, which can be treated as fractals and can be studied using appropriate mathematical tools. Capacitance and correlation fractal dimensions of largest gypsum caves of the Western Ukraine (as well as the World's largest ones) were calculated. The results were used to predict findings of new, undiscovered cave mazes parts.
\end{abstract}

Key words: fractals, gypsum caves, speleology, karst, Ukraine

\section{Introduction}

Natural objects possessing enough complicated spatial structure can be treated as fractals. What is important, calculations of so-called fractal dimensions can be performed in most typical cases. There are many different types of fractal dimensions named as: capacity, correlation, informative, topological, boxed, Hausdorff, Lyapunov, to mention widely used terms or synonyms. However, a common feature for all types of these fractal quantitative measures, is that the fractal dimension counts a self-similarity of an object at different spatial scales. In other words, a fractal dimension measures directly geometrical complexity of an object as a whole or additionally can be sensitive to uniformity of spatial distribution existing in a given object. Especially, a fractal dimension can keep information about surface roughness and edges complexity. Obviously, in order to perform proper analysis any information of interest should be collected in a form of an image for further numerical processing.

Regions and karst objects, including caves, usually have complicated spatial structure and possess a self-similarity property enabling treatment of them as fractals. Good examples of karst fractals are: the karst landscape densely dotted by craters and karst depressions, often overlapping each other, the corroded walls in caves covered by micro-forms, the rock massifs cut by nets of karsified fissures, and others structures. The problem is not discussed in details in scientific literature, there are only a few works devoted to fractal problematics in karst (Curl 1986, Laverty 1987, Kusumayudha et al. 2000, Finnesand, Curl 2009, Piccini 2011, Skoglund, Lauritzen 2011).

A specific example of a spatial, genetic, fractal-like organization are maze cave systems created in hypogenic circumstances forming enormous and dense nets of underground channels and corridors. Due to their spatial complexity such systems can be analyzed as fractal objects. Obviously, this fractal characteristics of labyrinthine caves - as specific natural objects - is interesting and this is a novelty in the field. However, the important question is: if such analysis has enough deep sense, which fractal dimension is optimally suitable for that purpose? Authors argue that this type of analysis makes sense and try to specify one of a possible field of application, namely, for prediction of existence of not discovered yet (not explored) parts of cave nets. This aspect of research has both theoretical importance, as well as, has practical meaning for speleologists trying to discover new unknown cave regions.

In opinion of authors, the mentioned above goal is optimally fulfilled by the use of capacity and correlation fractal dimensions. These dimensions characterize fractal geometrical complexity of objects and may indicate onto internal regularities, or onto level of heterogeneity providing information about a genetic complexity (monoor multi-factorial origin) manifested oneself as a specific spatial realization of mazes. Thus, a capacity dimension enables estimation of the general level of structural complexity, a variety of an object as a whole. The lower is its value, the greater is spatial multiplicity of a given cave (or its part). On the other hand, a correlation dimension 
additionally senses variations in a cave structural distribution. Additionally, an important source of information can come from a comparison between both fractal dimensions what will be discussed below.

\section{Studied objects - cave mazes}

Analytical studies were performed for four labyrinth caves chosen from the set (Fig. 1) of largest gypsum caves (in brackets a total channels length in $\mathrm{km}$ ): Optymistychna (188), Ozerna (111), Zoloushka (90), Kryshtaleva (22) (Klimchouk et al. 2009).

All the mentioned caves are located in West Ukraine (Podole and Bukowina regions) and are created in a Miocenic gypsum layer of 20-25 m thickness. The caves have similar hypogenic origin, were created in phreatic circumstances as a result of underground waters being under pressure acting on dense nets of fissures, located in gypsum, causing corrosion-like expansion of the latter. At the cave maps (Fig. 2), significantly extended fissures (up to dimensions of channels), created in different eras, and made by various geological mechanisms, are seen. Underground waters acting during speleogenesis, that is during penetration of features gypsum layer, caused diverse internal structures. These structures were formed (before speleogenesis) by subsequent overlapping of fracturing systems, of different origin, represented by lithogenetic and/or tectonic fissures, and importantly, represented by geometrical features (polygons, crosses, etc.). On the maps an uncommon complexity of nets roughness is visible. However, inside corridor webs, some regularities are easily distinguishable - the uniformities generated by fissure systems. For every cave, considered here, individual combinations (configurations) of overlapping polygons (lithogenetic) or crosses (tectonic) systems, influenced and extended by underground waters are distinguishable.

Using fractal analysis for quantitative description of complex caves, it might be possible, for the concrete cave, to estimate an initial role of the given fissure system during speleogenesis, or influence of any individual initial fissure systems onto evolution of the subsequent structural systems.

\section{Basic facts and research methodology}

Fractals dimensions, including capacity and correlation ones, are quantities describing in some situations normal figures, like lines, squares, cubes, providing normal integer values of these objects, that is: $1,2,3$, respectively. A capacity dimension is based on counting of unit-boxes covering an object (Fig. 3a, b). During a procedure boxes of down-scaled dimension are applied. The log-log dependence between number of boxes covering an object and a box size is linear within some range of variables. A capacity dimension is equal to a slope of that

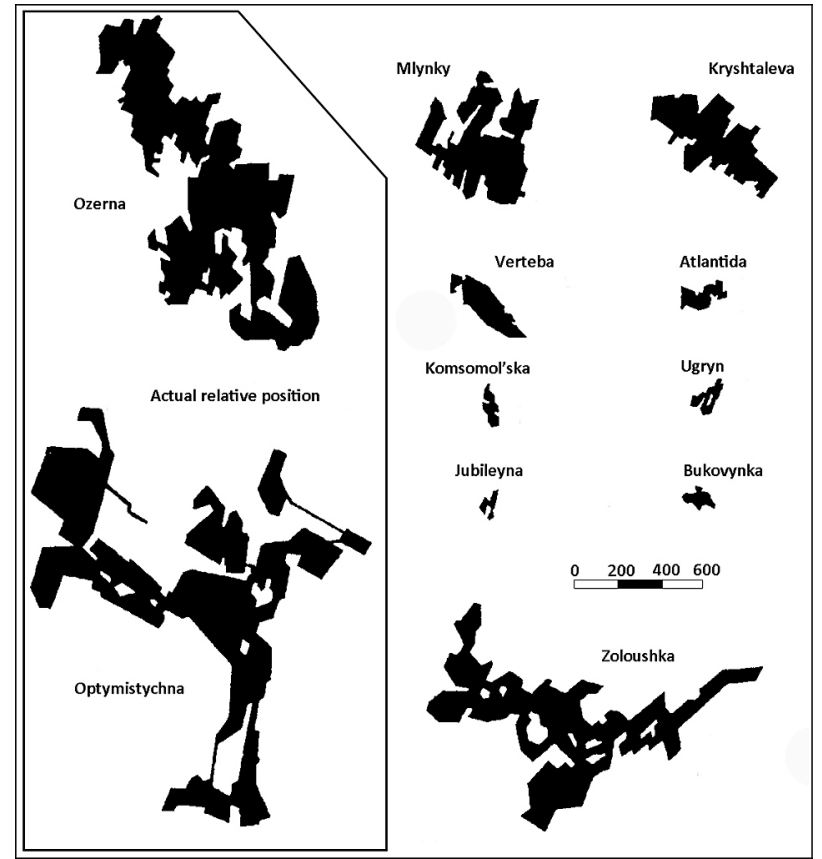

Fig. 1. Configurations and relative dimensions of some cave fields of the largest gypsum caves of Western Ukraine (including investigated caves). All contours are pictured at the same scale (Klimchouk et al. 2009)

linear dependence. A capacity dimension of a normal figure, like a triangle, equals 2 .

A correlation dimension methodology is similar to that of capacity dimension, as it is equal to a slope of linear log-log dependence between a correlation factor and unit-circles radii covering randomly chosen components of an object (Fig. 3c). If points in a 2-dimensional object, for example in a triangle, are distributed completely randomly, then the correlation dimension equals 2 . Importantly, a correlation dimension senses small-scale variations of an image, while a capacity dimension is not sensitive for local irregularities and represent uniquely an image as a whole (Baker, Gollub 1998, Peitgen et al. 2004).

Every dimension can be calculated from counting procedure of spatial unit objects of a length $\varepsilon$ covering the measured object of the length $L$ (Fig. 3a). If the procedure provides $N(\varepsilon)$ counted, squared objects (Fig. 3b), the capacity dimension can be calculated from the following expression

$$
L=N(\varepsilon) \cdot \varepsilon
$$

for a single dimensional object, or from the following formula

$$
L^{d_{c a p}}=N(\varepsilon) \cdot \varepsilon^{d_{c a p}},
$$

if the capacity dimension $d_{\text {cap }}$ is larger than 1 . Taking logarithms of Eq. 2 one obtains 
a)

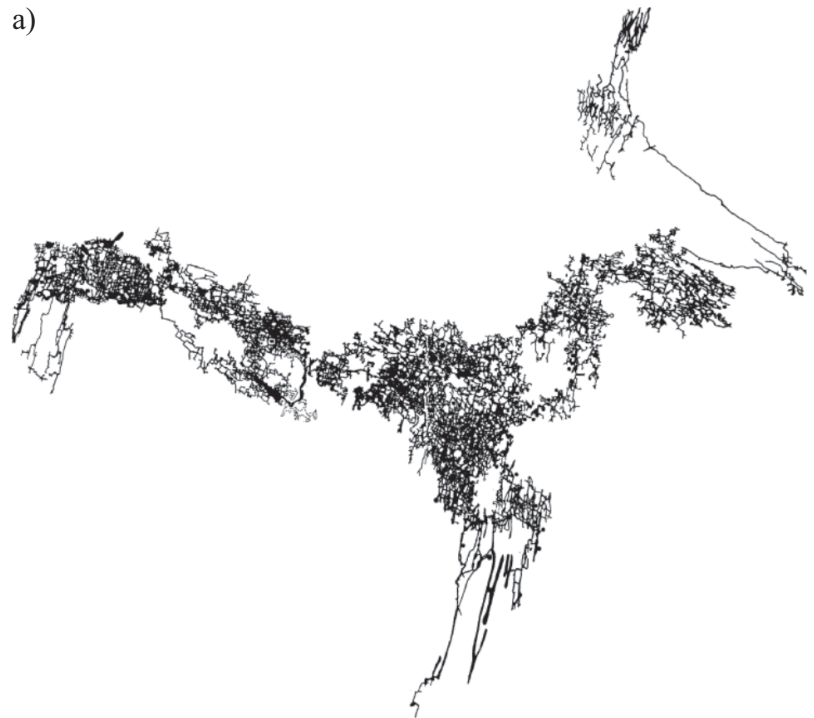

c)

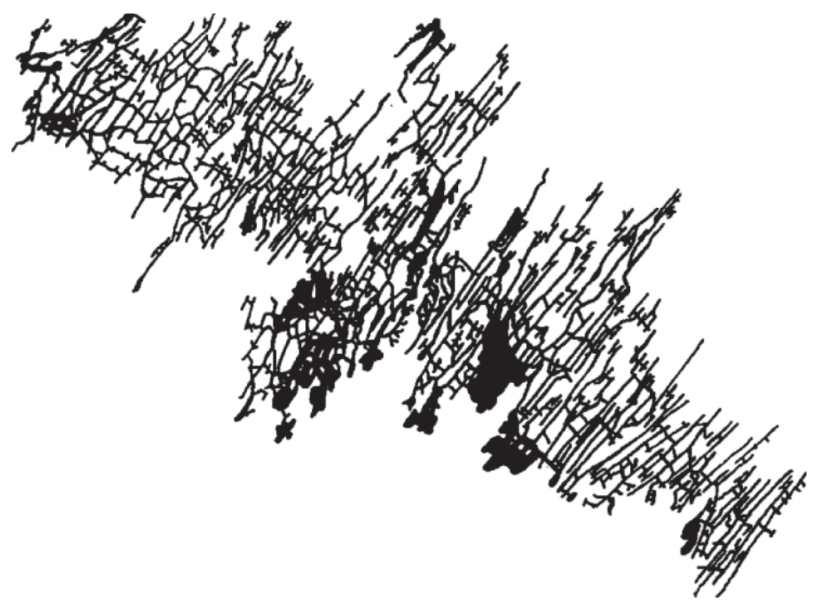

b)

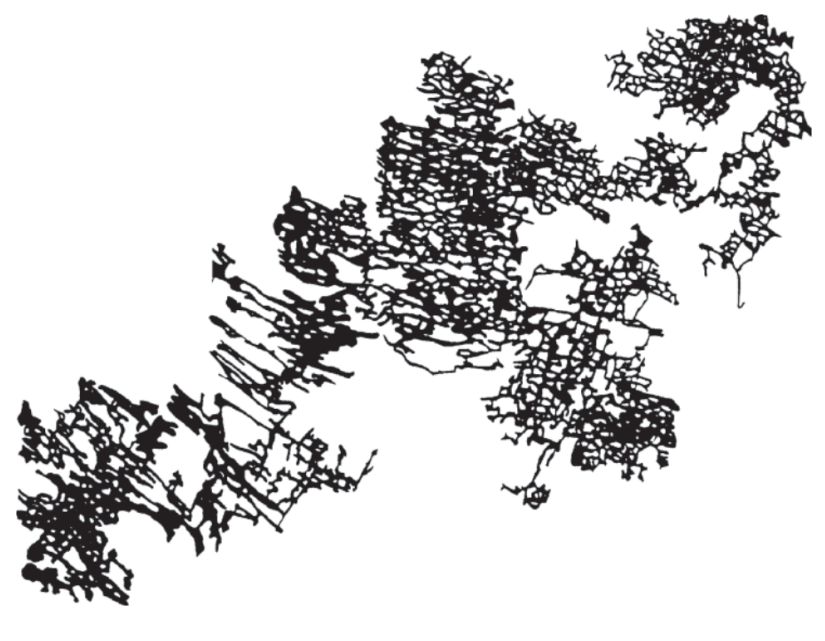

d)

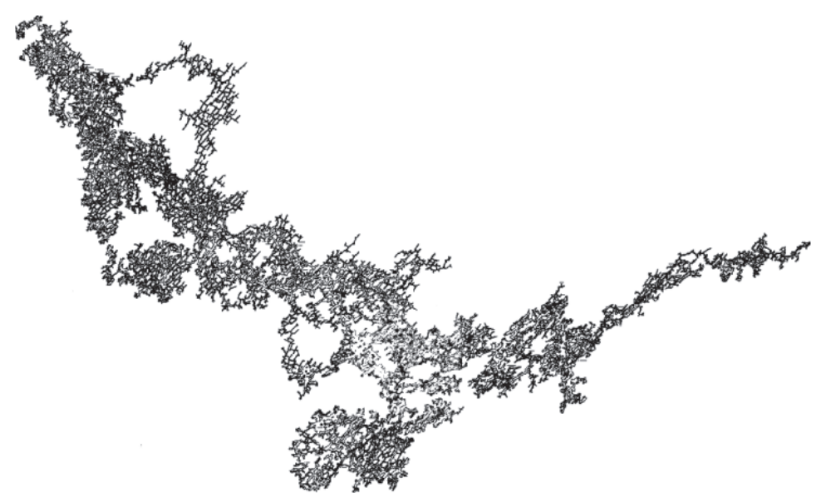

Fig. 2. Maps of caves: Optymistychna (a), Ozerna (b), Kryshtaleva (c), Zoloushka (d)

Note: spatial scales are different for the provided cases

$$
d_{\text {cap }}=\frac{\log (N(\varepsilon))}{\log (L)+\log ((1 / \varepsilon))} .
$$

In practice, the capacity dimension can be derived from a linear log-log dependence between number of boxes $N(\varepsilon)$ and the square size $\varepsilon$, being the fractional part $n$ of the analyzed size $L$. Thus, the slope of that dependence equals

$$
d_{\text {cap }} \approx \frac{\log (N(\varepsilon))}{\log ((1 / \varepsilon))}=\frac{\log (N(\varepsilon))}{\log ((n / L))}
$$

Another type of fractal dimension is the correlation one. That type of dimension employs a correlation factor, which counts mutual distances of randomly distributed points, lying on an analyzed object. Every point lies in a center of a circle of radius $R$ (Fig. 3c). For increasing radius the $C(R)$ factor grows, however for enough large $\mathrm{R}$-values the factor saturates since analyzed region can be completely included and covered by circles. The correlation factor is defined as follows

$$
C(R)=\frac{1}{N^{2}} \sum_{i=1}^{i=N} \sum_{\substack{j=1 \\ j \neq i}}^{j=N} H\left[R-\left|x_{i}-x_{j}\right|\right]
$$

where $N$ is the number of points, and $H\left[R-\left|x_{i}-x_{j}\right|\right]$ is the Heaviside step function

$$
H\left[R-\left|x_{i}-x_{j}\right|\right]=\left\{\begin{array}{lll}
1 & \text { if } & \left(R-\left|x_{i}-x_{j}\right|\right)>0 \\
0 & \text { if } & \left(R-\left|x_{i}-x_{j}\right|\right)<0
\end{array}\right.
$$

Since the correlation factor is proportional to a radius, $C(R)=$ const $\cdot R^{d_{c o r}}$, via the correlation dimension $d_{c o r}$, then the latter can be calculated from the following expression

$$
d_{\text {cor }}=\frac{\log (C(R))}{\log (R)}-\frac{\log (\text { const })}{\log (R)}
$$


and the dimension can be, in practice, calculated from the following expression

$$
d_{c o r} \approx \frac{\log (C(R))}{\log (R)},
$$

that is, can be derived from the linear log-log dependence between corresponding values.

\section{Interpretation of results and conclusions}

Performed image analysis of mono-colored maps enabled calculations of the capacity fractal dimension (Fig. 4 ) and the correlation fractal dimension (Fig. 5). Final results are given in tables 1 and 2 .

The most fractal-like character has Optymistychna cave - the capacity and correlation dimensions are significantly different from other three cases - since calculated values of the capacity dimension, and the correlation dimension are equal to 1.71 , and 1.76 , respectively, and are the relatively smallest values for the considered caves. That fact shows onto a relatively more complex general geometrical structure. From a geomorphological point of view, it indicates also onto the significant participation in speleogenesis of both lithogenetic and tectonic factors associated with polygonal and crossing-like fissures systems. Significantly less complicated structure of Zoloush$\mathrm{ka}$ and other caves indicates onto domination of one genetic factor (lithogenic or tectonic), which made a shape somehow more ordered. The regular features, represented by dominating number of passages, are clearly noticeable in Ozerna cave (in chosen parts) and in Kryshtaleva cave (as a whole).

This conclusion is confirmed by values of correlation dimension, which is sensitive for structure uniformity. Also, what is normal, it is slightly higher than that of a capacity one. From that perspective, the smallest correlation dimension of the Optymistychna cave (1.76) indicates onto larger spatial irregularities in a structure that in the Kryshtaleva cave (1.83), what is clearly visible in provided pictures.

Also, as it was mentioned, the important meaning for a quantitative description have a difference between capacity and correlation dimensions. In general, a larger value of a correlation dimension, in respect to a capacity one, thus existence of a difference between these dimensions, is something normal, since it results from mathematical structure of calculations, is natural for most dynamical systems and possesses geometrical origin. However, comparable values, or even equal ones, might suggest that normal rules are somehow deviated, thus it can inform about aberrations from a fractal mechanism characterizing a building structure. In a spatio-structural language this can mean that some parts of cave are not yet discovered or, at least, not included in graphical charts. Just from this hypothesis results a predictive importance a)

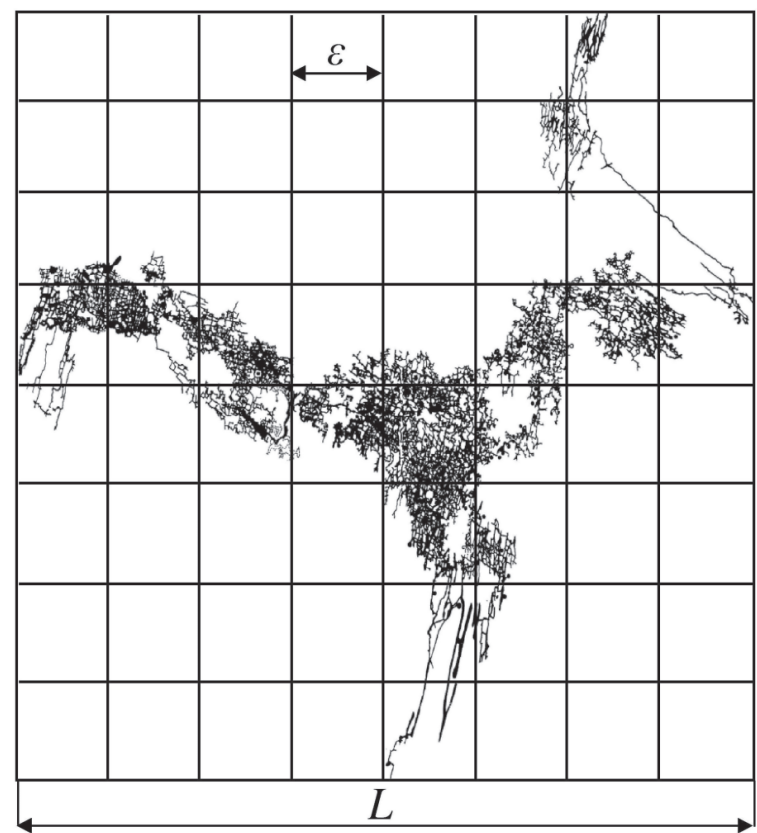

b)

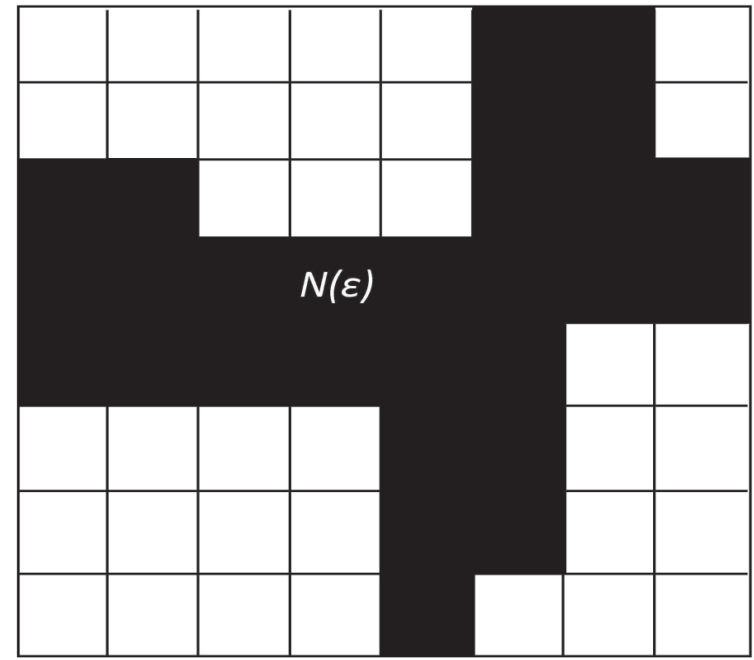

c)

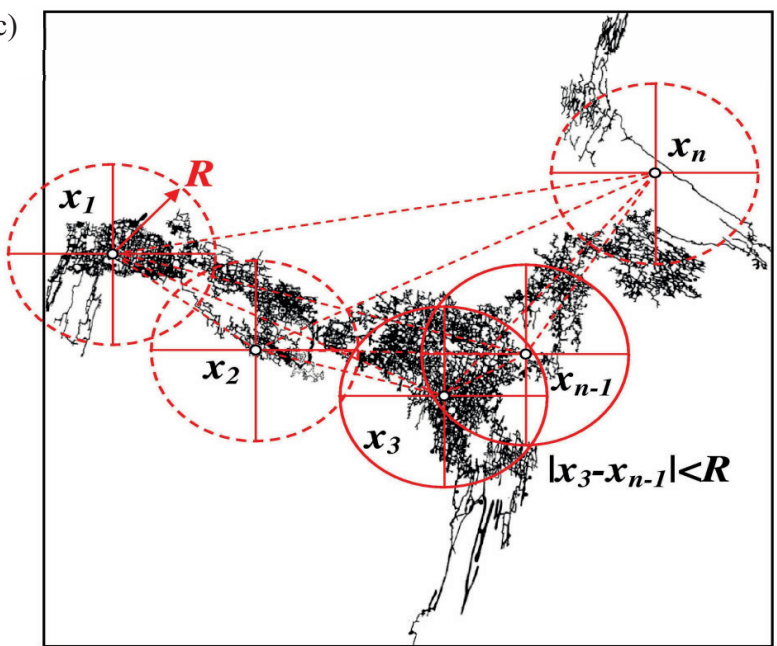

Fig. 3. An explanation of principles leading to capacity $(a, b)$ and correlation (c) fractal dimensions

of a comparative analysis of both dimensions. How much it is correct, that will be revealed by future speleological investigations of caves. 

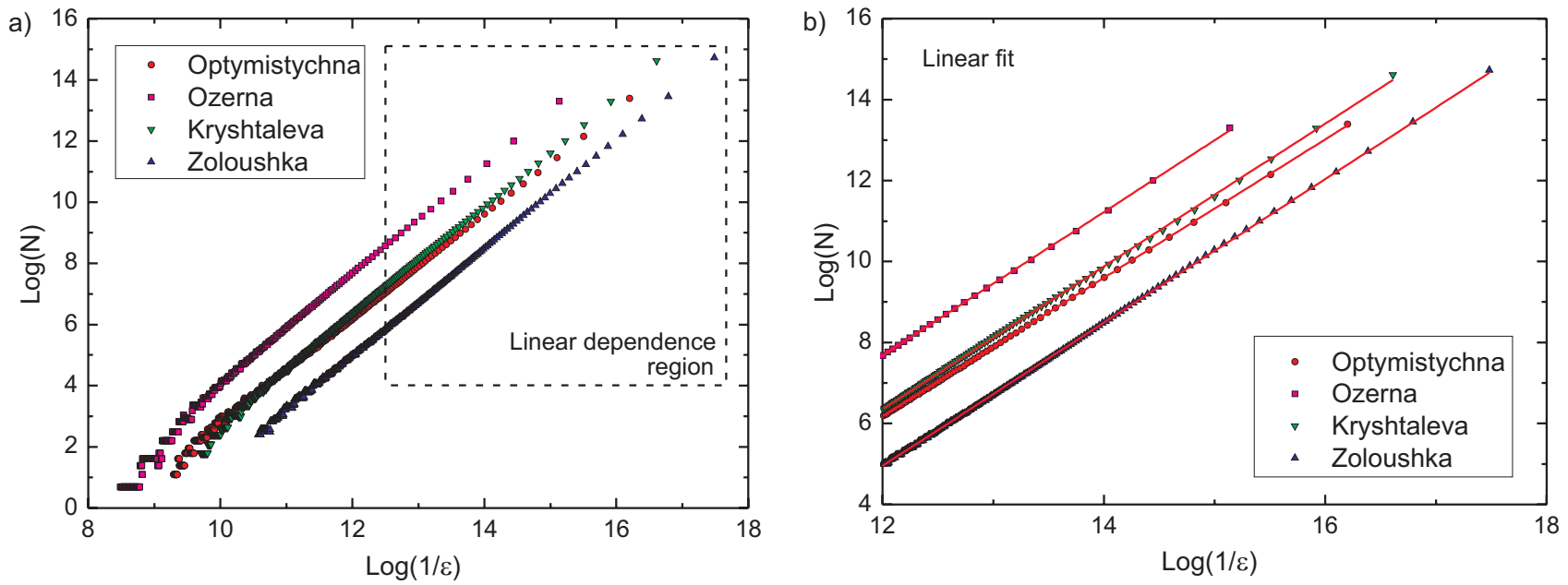

Fig. 4. The dependence between number of boxes covering analyzed pictures of caves and a box dimension (a). The capacity dimensions can be determined from linear fitting to linear dependence regions (b)
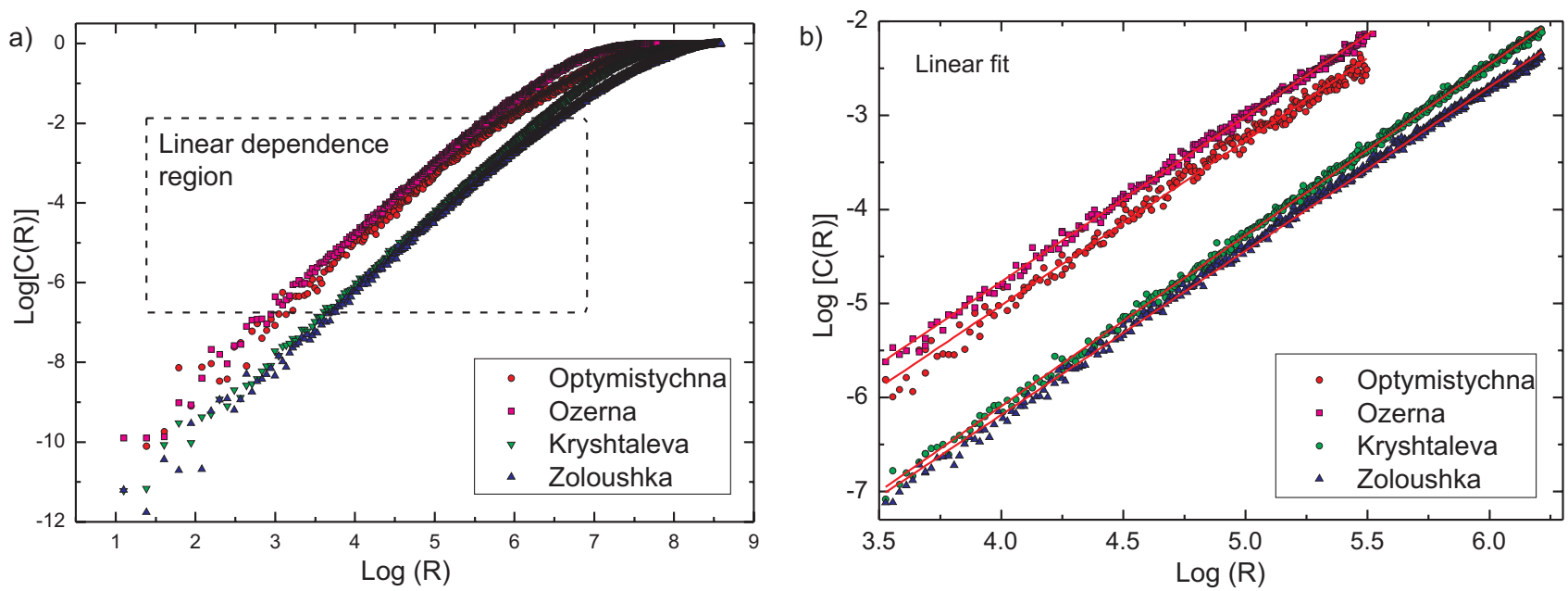

Fig. 5. The dependence between correlation factor and radii of circles associated with randomly distributed points representing caves (a). The correlation dimensions can be determined from fitting using linear regression (b)

Table 1. Summary of results for studied caves

\begin{tabular}{lcccc}
\multicolumn{1}{c}{ Cave } & Capacity dimension $d_{\text {cap }}$ & Uncertainty of $d_{\text {cap }}$ & Correlation dimension $d_{\text {corr }}$ & Uncertainty of $d_{\text {corr }}$ \\
\hline Optymistychna & 1.71 & 0.02 & 1.76 & 0.03 \\
\hline Ozerna & 1.78 & 0.03 & 1.79 & 0.03 \\
\hline Kryshtaleva & 1.76 & 0.03 & 1.83 & 0.03 \\
\hline Zoloushka & 1.76 & 0.02 & 1.80 & 0.03 \\
\hline
\end{tabular}

Thus, from the presented point of view, Ozerna cave really stands out. Thus, looking onto cave picture, and taking into an account the fact that the correlation and the capacity dimensions are comparable (the difference equals 0.01), it might indicate onto not discovered yet parts, which should complete structural morphology and increase correlation dimension to the higher value of

Table 2. Spatial scales for pixels in analyzed images

\begin{tabular}{lcc} 
Cave & $\begin{array}{c}\text { Pixel size } \\
(\mathrm{m})\end{array}$ & $\begin{array}{c}\text { Picture dimension } \\
\text { (pixels) }\end{array}$ \\
\hline Optymistychna & 2.22 & $3295 \times 2952$ \\
\hline Ozerna & 2.22 & $1936 \times 1437$ \\
\hline Kryshtaleva & 0.39 & $4048 \times 2983$ \\
\hline Zoloushka & 2.22 & $6263 \times 3749$ \\
\hline
\end{tabular}

about $0.05-0.07$, under the assumption, that a difference between both dimensions is a solid rule for caves. A larger difference and smaller "reservoir" for undiscovered parts has Zoloushka (the difference equals 0.02-0.04), next Optymistychna cave $(0.03-0.05)$, a finally the smallest possibility for undiscovered part might reveal Kryshtaleva cave (0.04-0.07).

As a curiosity of described caves we would like to present a hypothetical cave, with no internal structure, possessing a single compact volume, derived graphically from Ozerna cave (Fig. 6.). For this case, both the capacity and correlation dimensions are now grater, more closer to the numerical value of 2 , and both the dimension are equal within the obtained accuracy of calculations. 


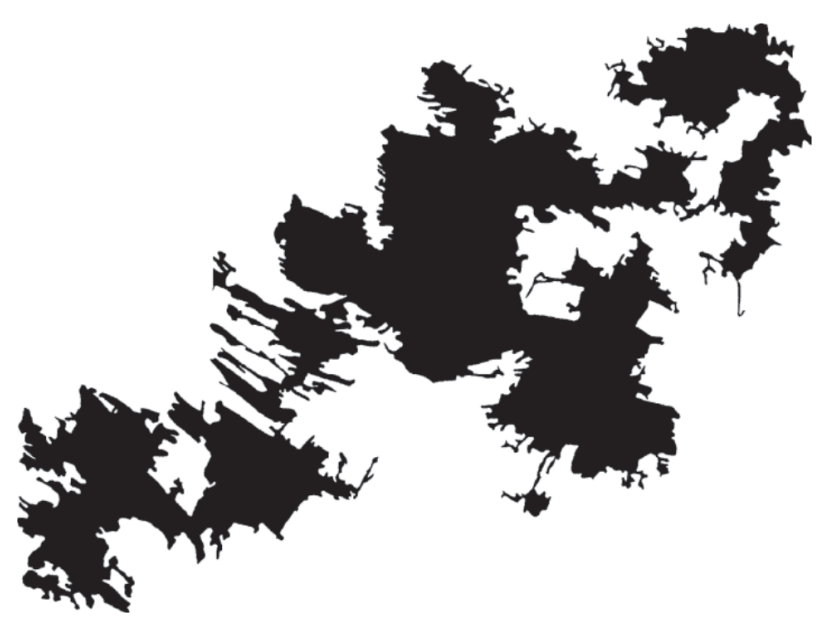

Fig. 6. Hypothetical cave derived from Ozerna Cave. Its fractal dimensions are equal to $1.84 \pm 0.02$, and $1.84 \pm 0.02$, for the capacity dimension and correlation dimension, respectively

All analyzed caves can be treated like fractals and their capacity and correlation fractal dimension were calculated. It is a hope of authors, that presented calculations of fractal dimensions provided a lot of information, which interpreted from this methodology perspective, will support future speleomorphologic and speleogenetic investigations.

\section{References}

Baker G.L., Gollub J.P., 1998. Chaotic Dynamics: An Introduction. Cambridge University Press.

Curl R.L., 1986. Fractal dimensions and geometries of caves. Mathematical Geology 18(8): 765-783. http://dx.doi.org/10.1007/BF00899743

Finnesand T., Curl R.L., 2009. Morphology of Tjoarvekrajgge, the longest cave of Scandinavia. Speleogenesis. Proc. 15th International Congress of Speleology, Kerrville, Texas, vol. 2: 878-883.

Klimchouk A., Andreychouk V., Turchinov I., 2009. The structural prerequisites of speleogenesis in gypsum in the Western Ukraine. University of Silesia-Ukrainian Institute of Speleology and Karstology. Sosnowiec-Symferopol: $96 \mathrm{pp}$.

Kusumayudha S.B., Zen M.T., Notosiswoyo S., Gautama R.S., 2000. Fractal analysis of the Oyo river, cave system and topography of the Gunungsewu karst area, central Java, Indonesia. Hydrogeology Journal 8: 271-278.

http://dx.doi.org/10.1007/s100400050014

Laverty M., 1987. Fractals in karst. Earth Surface Processes and Landforms 12(5): 475-480. http://dx.doi.org/10.1002/esp.3290120505

Peitgen H.O., Jürgens H., Saupe D., 2004. Chaos and Fractals: New Frontiers of Science. Springer.

Piccini L., 2011. Recent developements on morphometric analysis of karst caves. Acta Carsologia 40(1): 43-52.

Skoglund R.O., Lauritzen S.E., 2011. Subglacial maze origin in low-dip marble stripe karst: examples for Norway. Journal of Cave and Karst Studies 73(1): 31-43.

http://dx.doi.org/10.4311/jcks2009ES0108 